\title{
Approximating the Time-Frequency Representation of Biosignals with Chirplets
}

\author{
Omid Talakoub, Jie Cui, and Willy Wong \\ Department of Electrical and Computer Engineering, University of Toronto, On, Canada M5S 1A1 \\ Correspondence should be addressed to Willy Wong, willy@eecg.utoronto.ca
}

Received 14 January 2010; Accepted 29 April 2010

Academic Editor: Syed Ismail Shah

Copyright ( $\odot 2010$ Omid Talakoub et al. This is an open access article distributed under the Creative Commons Attribution License, which permits unrestricted use, distribution, and reproduction in any medium, provided the original work is properly cited.

\begin{abstract}
A new member of the Cohen's class time-frequency distribution is proposed. The kernel function is determined adaptively based on the signal of interest. The kernel preserves the chirp-like components while removing interference terms generated due to the quadratic characteristic of Wigner-Ville distribution. This approach is based on the chirplet as an underlying model of biomedical signals. We illustrate the method using a number of common biological signals including echo-location and evoked potential signals. Finally, the results are compared with other techniques including chirplet decomposition via matching pursuit and the Choi-Williams distribution function.
\end{abstract}

\section{Introduction}

Many signals of biological origin are nonstationary in nature. Examples include speech signals, bat calls as well as neuroelectric signals like electroencephalography (EEG) $[1,2]$, heart rate variability [3], or event-related potentials (ERPs) [4]. Time-frequency or time-scale representations, in recent years, have found significant application in nonstationary analysis of a wide-range of signals including biomedical signals [5-13]. Constructing a time-frequency representation involves mapping a one-dimensional time-domain signal $x(t)$ into a two-dimensional function of time and frequency or time and scale [14]. Time-frequency representations are some of the main tools for nonparametric instantaneous frequency estimation [14]. The position of peaks in the timefrequency representation reveals the main components or structures of the signal.

Among the most commonly used time-frequency distributions are the so-called quadratic distributions. The spectrogram $[15,16]$ is one of the earliest proposed distributions yet is still commonly used to this day. Nevertheless, the spectrogram has severe drawbacks, both theoretically since it provides biased estimators of the signal instantaneous frequency and group delay [17], and practically since the Gabor-Heisenberg inequality [15] makes tradeoffs between temporal and spectral resolution unavoidable. To overcome these shortcomings, other nonstationary representations have been proposed. Among these include the Cohen's class [18] of bilinear time-frequency energy distributions. The Wigner-Ville distribution [19], the Margenau-Hill distribution [20], their smoothed versions [21-23], and others with reduced cross-terms [24-27] are all members of this class. Although Cohen's class distributions tend to reduce the interference between the various signal subcomponents, this reduction can affect the precision by which the instantaneous frequency is estimated. This is mainly due to the predefined smoothing kernel functions which do not distinguish between the signal components and the interference terms. Hence, in the process of reducing or removing crossterms, the kernel also removes signal components. On the contrary, signal-dependent kernels can provide improved time-frequency representation and have been proposed for various applications [28-31]. An extensive review of the methods proposed for improving time-frequency resolution can be found in [14].

The nonparametric methods of time-frequency analysis described above can be contrasted with parametric approaches which attempt to model the underlying signal $[32,33]$. There has been much debate as to the ideal choice of basis functions to use. Generally speaking, the 
more similar the basis function is to the signal, the more compact is the decomposition. Many biological signals can be thought of as a sum of more elementary components each of which are relatively narrowband in nature. Common examples include speech which consist of a number of formant frequencies illustrating the resonance of the vocal tract. In such a case, chirplets (or chirp signals of limited time extent) can be thought of as a good model of the underlying signal-any narrowband changes in instantaneous frequency can be described mathematically to first order by linear changes in the time-frequency plane [34-36]. We have been working on ways to decompose biological signals into a sum of chirplets [37]. A time-frequency representation can be obtained from the decomposition by summing up the individual contributions from each chirplet. This provides a clear time-frequency picture of the signal without the crossterm interference. While we have found that this method yields excellent visualization of biomedical signals, there are some significant challenges to overcome because chirplets do not form an orthogonal basis set. In some earlier work, we used matching pursuit to carry out the decomposition process which we found to be prohibitive in terms of computational cost. There is a need to find improved ways to carry out this analysis.

This paper proposes a new class of time-frequency distributions for which the kernel function is determined adaptively based on the signal of interest. This approach can be best characterized as a hybrid approach combining both nonparametric and parametric methods using the chirplet as an underlying model of the biomedical signal. The kernel function preserves the chirping components in the signal while eliminating the interference terms generated by the quadratic characteristic of the time-frequency representation. The proposed method filters out the oscillatory crossterms and instead preserves the "true" signal components which are of low spatial frequency.

\section{Proposed Method}

2.1. Wigner-Ville Distribution and Multicomponent Signals. Time-frequency representations via the wavelet [38], windowed Fourier transforms and chirplet transform [39] are computed by correlating the signal with a family of timefrequency atoms. The time-frequency resolution of the distributions is therefore limited by the resolution of these atoms. In contrast, the Wigner-Ville Distribution (WVD) defines signal energy density in time-frequency plane with no restriction on resolution beyond the uncertainty principle. The WVD is computed by correlating the signal with a time and frequency translation of itself [40]:

$$
W V_{f}(t, \omega)=\int_{-\infty}^{\infty} f\left(t+\frac{\tau}{2}\right) f^{*}\left(t-\frac{\tau}{2}\right) e^{-i \omega \tau} d \tau .
$$

Due to the quadratic nature of the distribution, the application of the Wigner-Ville distribution is limited by the existence of interference terms. The interference can be best illustrated by considering multicomponent signals. We can think of a multicomponent signal $f(t)$ as a sum of more elementary monocomponents, $f(t)=\sum f_{k}(t)$. In Section 2.2, we will explore the specific case where the monocomponents are Gaussian chirplet functions. The WVD of a multicomponent signal consists of the summation of auto- and interference terms (cross-terms) due to pairwise interaction of components:

$$
\begin{aligned}
& W V_{f}(t, \omega) \\
& =\sum_{k} W V_{f, k}+\sum_{n \neq m} \sum_{m} \int_{-\infty}^{\infty} f_{n}\left(t+\frac{\tau}{2}\right) f_{m}^{*}\left(t-\frac{\tau}{2}\right) e^{-i \omega \tau} d \tau,
\end{aligned}
$$

where $W V_{f, k}$ is WVD of the $k$ th monocomponent autoterm. Cross-terms may lead to an erroneous visual interpretation of the time-frequency representation and are also a hindrance to pattern detection, since the interference can overlap with the signal. Due to the marginal properties of the WVD, i.e., $\int W V_{f}(t, \omega) d t=|F(\omega)|^{2}$, and $\int W V_{f}(t, \omega) d \omega=$ $2 \pi|f(t)|^{2}$, the interference terms are oscillatory and zeromean if the individual components do not overlap at any point in time and frequency [40]. The spatial frequency of the oscillations depends on the distance between the monocomponents in time-frequency plane; that is, the farther apart the components, the higher the oscillation frequency. Although these interferences can be attenuated by time-frequency averaging, this will result in the loss of energy localization.

The Cohen's class distribution extends the Wigner-Ville distribution by introducing a smoothing kernel [18]:

$$
W V_{f, \theta}=\iint_{-\infty}^{\infty} W V_{f}(\tau, \zeta) \theta(t-\tau, \omega-\zeta) d \tau d \zeta
$$

Since convolutions can be more easily manipulated in the transformed space, a two-dimensional Fourier transform of $W V_{f}(t, \omega)$ with respect to $t$ and $\omega$ yields what is known as the ambiguity function. Based on (2), the ambiguity function of a multicomponent signal can be expressed in terms of the summation of two-dimensional Fourier transformation of monocomponents and cross-terms:

$$
A_{s}\left(\Omega_{1}, \Omega_{2}\right)=\sum_{k=1}^{N} A_{c}^{k}\left(\Omega_{1}, \Omega_{2}\right)+I\left(\Omega_{1}, \Omega_{2}\right),
$$

where $A_{c}^{k}\left(\Omega_{1}, \Omega_{2}\right)$ is the ambiguity function of $k$ th monocomponent and $I\left(\Omega_{1}, \Omega_{2}\right)$ the ambiguity function of the interference terms. While it is not always possible to express $I\left(\Omega_{1}, \Omega_{2}\right)$ in closed form, one can always work with the expression numerically. The transform of (3) gives the multiplication of the signal's ambiguity function with the transform of the kernel. That is, $A_{s, \theta}\left(\Omega_{1}, \Omega_{2}\right)=$ $A_{s}\left(\Omega_{1}, \Omega_{2}\right) \cdot A_{\theta}\left(\Omega_{1}, \Omega_{2}\right)$. An ideal kernel should preserve each individual component and its localization in time-frequency domain while removing the cross-terms, that is, $A_{s}\left(\Omega_{1}, \Omega_{2}\right)$. $A_{\theta}\left(\Omega_{1}, \Omega_{2}\right)=\sum_{k=1}^{N} A_{c}^{k}\left(\Omega_{1}, \Omega_{2}\right)$.

2.2. The Wigner-Ville and Ambiguity Representation with Gaussian Chirplets. Next we consider the specific case where 
the monocomponents of a multicomponent function are approximated by Gaussian chirplets.

The chirp is one of the most fundamental signals in nature. Many natural and man-made signals can be well approximated using chirps including seismological signals, radar systems, evoke potentials [37], ultrasound signals $[41,42]$, and marine-mammal signals [43, 44]. A Gaussian chirplet is a component whereby its instantaneous frequency changes linearly over time and is localized in time by a Gaussian envelop. A normalized Gaussian chirplet is defined in the time domain as

$$
\begin{aligned}
c(t)= & \left(\frac{\alpha}{\pi}\right)^{1 / 4} \exp -\left\{\frac{\alpha\left(t-t_{0}\right)^{2}}{2}\right\} \\
& \times \exp \left\{j\left[\omega_{0}+\frac{\beta}{2}\left(t-t_{0}\right)\right]\left(t-t_{0}\right)\right\},
\end{aligned}
$$

where $\alpha>0$ is time spread of the signal, $t_{0}$ is center of time, $\omega_{0}$ is center of frequency, and $\beta$ is the chirp rate [39]. $f(t)$ is normalized to have unit energy. The Wigner-Ville distribution of $f(t)$ can be expressed as

$$
\begin{aligned}
W V_{c}(t, \omega)= & 2 \exp \left\{-\alpha\left(t-t_{0}\right)^{2}\right\} \\
& \times \exp \left\{-\frac{1}{\alpha}\left[\left(t-t_{0}\right) \beta-\left(\omega-\omega_{0}\right)\right]^{2}\right\} .
\end{aligned}
$$

Furthermore, it is notable that when $\alpha \rightarrow 0$ the chirplet becomes a chirp, $e^{j\left[\omega_{0}+(\beta / 2)\left(t-t_{0}\right)\right]\left(t-t_{0}\right)}$. Hence the WVD of a chirp becomes

$$
\lim _{\alpha \rightarrow 0} W V_{c}(t, \omega)=2 \pi \delta\left[\left(t-t_{0}\right) \beta-\left(\omega-\omega_{0}\right)\right]
$$

which shows a precise localization of instantaneous frequency and energy. Note however that this is not the case if the changes in instantaneous frequency are not linear $[14,45]$.

The ambiguity function of a Gaussian chirplet is expressed as

$$
\begin{aligned}
F\left\{W V_{c}(t, \omega)\right\}= & A_{c}\left(\Omega_{1}, \Omega_{2}\right)=2 \pi \exp \left\{-\frac{\left(\Omega_{1}-\beta \Omega_{2}\right)^{2}}{4 \alpha}\right\} \\
& \times \exp \left\{-\frac{\alpha\left(\Omega_{2}\right)^{2}}{4}\right\} \exp \left\{-j\left(\Omega_{1} t_{0}-\Omega_{2} \omega_{0}\right)\right\} .
\end{aligned}
$$

It should be noted that the ambiguity function of a Gaussian chirplet is a zero-mean bivariate Gaussian density with covariance matrix determined by the time spread $(\alpha)$ and the chirp rate $(\beta)$. Due to the oscillatory nature of the cross-terms, the interference is located away from the origin $[25,33]$. For instance, consider the signal $g(t)$ which is equal to the sum of two chirplets, $g(t)=A_{1} c_{1}\left(t, \alpha, \beta, t_{1}, \omega_{1}\right)+$ $A_{2} c_{2}\left(t, \alpha, \beta, t_{2}, \omega_{2}\right)$. The WVD of $g(t)$ is expressed as

$$
\begin{aligned}
& W V_{g}(t, \omega) \\
& =\left|A_{1}\right|^{2} W V_{c}\left(t, \omega, \alpha, \beta, t_{1}, \omega_{1}\right) \\
& +\left|A_{2}\right|^{2} W V_{c}\left(t, \omega, \alpha, \beta, t_{2}, \omega_{2}\right) \\
& +2 \operatorname{Re}\left\{A_{1} A_{2}^{*} W V_{c}\left(t, \omega, \alpha, \beta, \frac{t_{1}+t_{2}}{2}, \frac{\omega_{1}+\omega_{2}}{2}\right)\right. \\
& \left.\quad \times e^{j\left[(t)\left(\omega_{1}-\omega_{2}\right)-\left(\omega-\left(\omega_{1}+\omega_{2}\right) / 2\right)\left(t_{1}-t_{2}\right)\right]}\right\},
\end{aligned}
$$

where $W V_{c}(t, \omega)$ is defined in (6). The ambiguity function is

$$
\begin{aligned}
I\left(\Omega_{1}, \Omega_{2}\right) \\
=\left(4 \pi e^{-\left(\Omega_{1}-\beta \Omega_{2}\right)^{2} / 4 \alpha} e^{-\alpha\left(\Omega_{2}\right)^{2} / 4} e^{-j\left[\Omega_{1}\left(\left(t_{1}+t_{2}\right) / 2\right)+\Omega_{2}\left(\left(\omega_{1}+\omega_{2}\right) / 2\right)\right]}\right) \\
\quad *\left(\delta\left(t_{1}-t_{2}, \omega_{1}-\omega_{2}\right)+\delta\left(t_{2}-t_{1}, \omega_{2}-\omega_{1}\right)\right),
\end{aligned}
$$

where $\delta(t, \omega)$ is a two dimensional Dirac delta function, and “*” denotes the two-dimensional convolution operator. The above equation shows that the interferences are concentrated at $\left(t_{1}-t_{2}, \omega_{1}-\omega_{2}\right)$ and $\left(t_{2}-t_{1}, \omega_{2}-\omega_{1}\right)$ with the autoterms near the origin. Please see Figure 1. This example can be generalized to a sum of any number of chirplets with arbitrary parameters and proves for the general case that the interference terms are located away from the origin [46]. This observation holds important application for the determination of the adaptive kernel to be discussed in the next section.

2.3. Optimal Kernel Determination. Equal density contours for the autoterms of chirplets are defined mathematically by ellipsoids. The direction and length of the principle axes are functions of the chirp rate and the time spread. These axes can be identified with a Radon transform. Analysis of the Radon transform reveals information regarding the monocomponent chirp rates $(\beta)$ and time spreads $(\alpha)$. Recall that the chirplet components lie at the origin of the ambiguity space while the interference terms are located away from the origin. The Radon transform of ambiguity function of a normalized chirplet can be expressed as

$$
\begin{aligned}
& R_{c}(\rho=0, \theta) \\
& =\iint_{-\infty}^{\infty} A_{c}\left(\Omega_{1}, \Omega_{2}\right) \delta\left(\Omega_{1} \cos \theta+\Omega_{2} \sin \theta\right) d \Omega_{1} d \Omega_{2} \\
& \quad=2 \pi \sqrt{\frac{\pi}{\lambda(\theta)}} \exp \left\{-\frac{\left[\omega_{0}-t_{0} \tan \theta\right]^{2}}{4 \lambda(\theta)}\right\},
\end{aligned}
$$

where $\lambda(\theta)=(\beta+\tan \theta)^{2} / 4 \alpha+\alpha / 4$. Based on the superposition property, the Radon transform will show peaks at values of $\theta$ corresponding to the axes orientations of each of the ellipsoids. In order to exclude the effect of the interference terms in the calculation, the Radon transform is carried out 


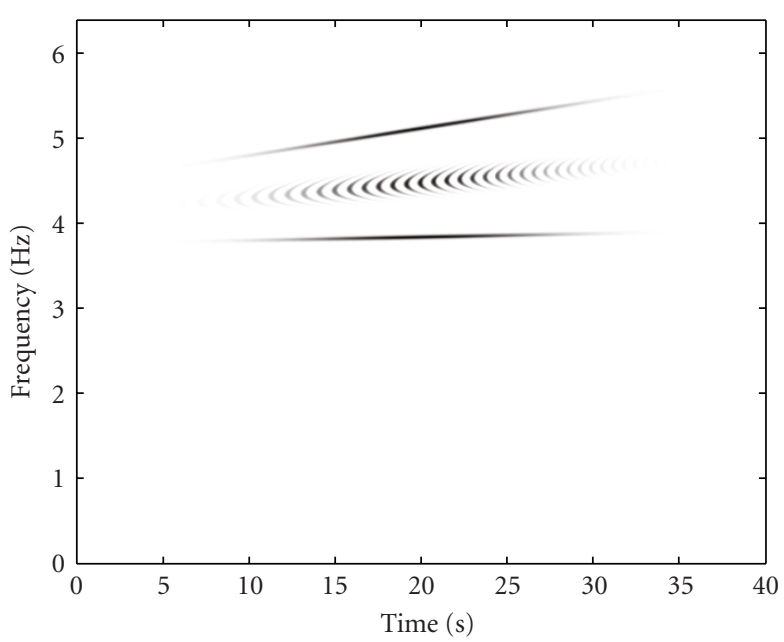

(a)

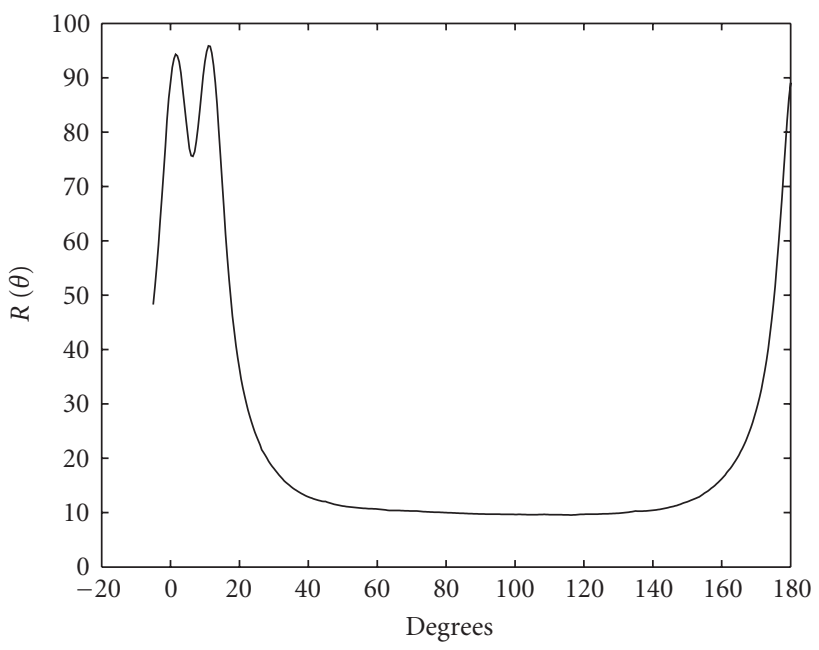

(c)

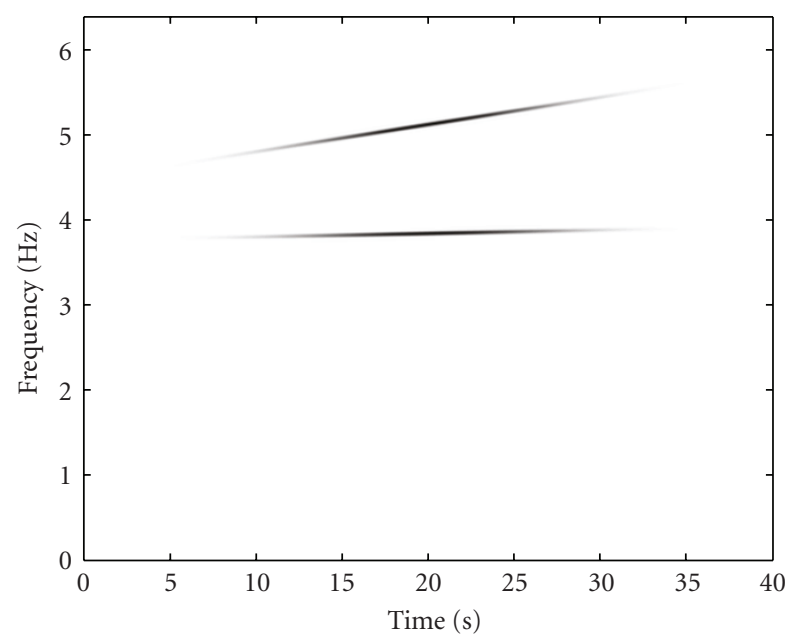

(e)

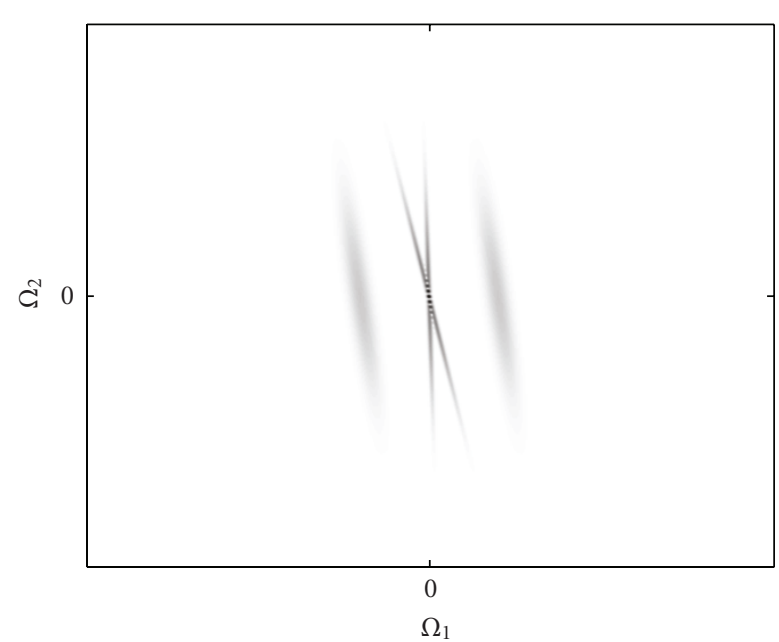

(b)

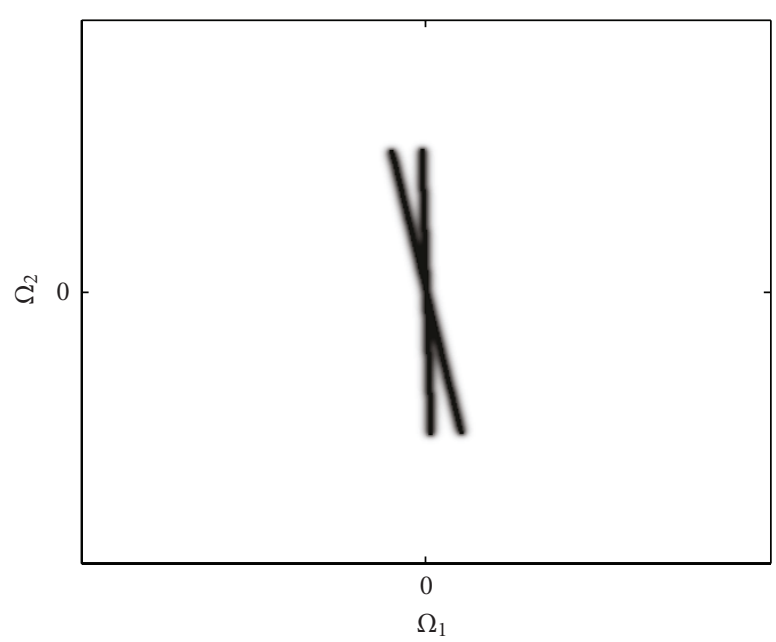

(d)

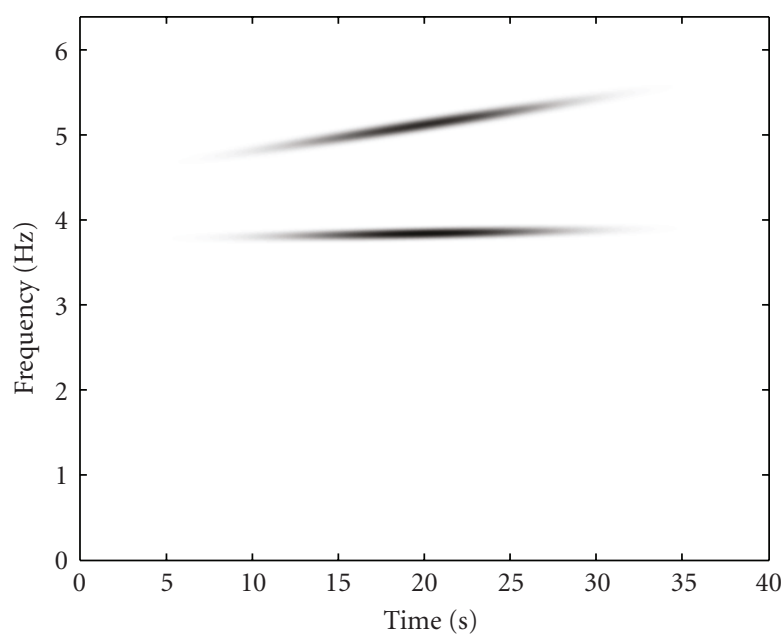

(f)

FIGURE 1: (a) WVD of two chirplets $\left(\alpha_{1}=0.01, \beta_{1}=0.2, t_{1}=20.05, \omega_{1}=32.08, \alpha_{2}=0.01, \beta_{2}=0.025, t_{1}=20.05\right.$, and $\left.\omega_{2}=24.06\right)$. (b) Representation of chirplets in ambiguity space. Cross-terms are located between the chirplets in the WVD, while in ambiguity space they are located away from the origin. (c) Radon transformation in the neighbourhood of the origin $(\rho=0)$. (d) Optimal kernel in the ambiguity space. (e) Resulting time-frequency representation. (f) Smoothed pseudo-Wigner-Ville distribution of the signal. Reduction in energy localization is noticeable in this representation. 


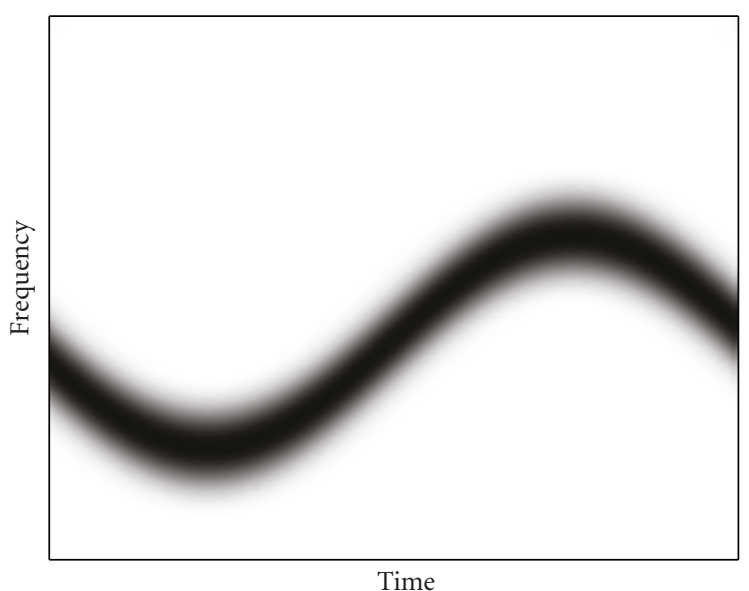

(a)

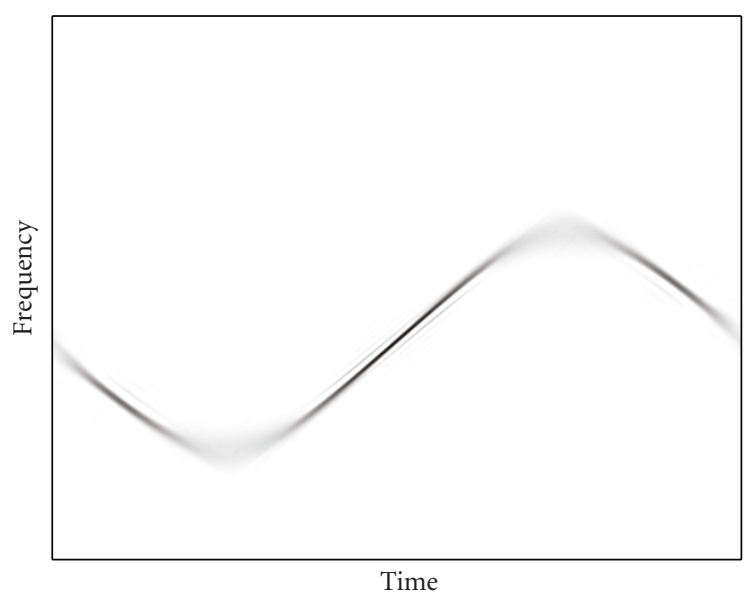

(c)

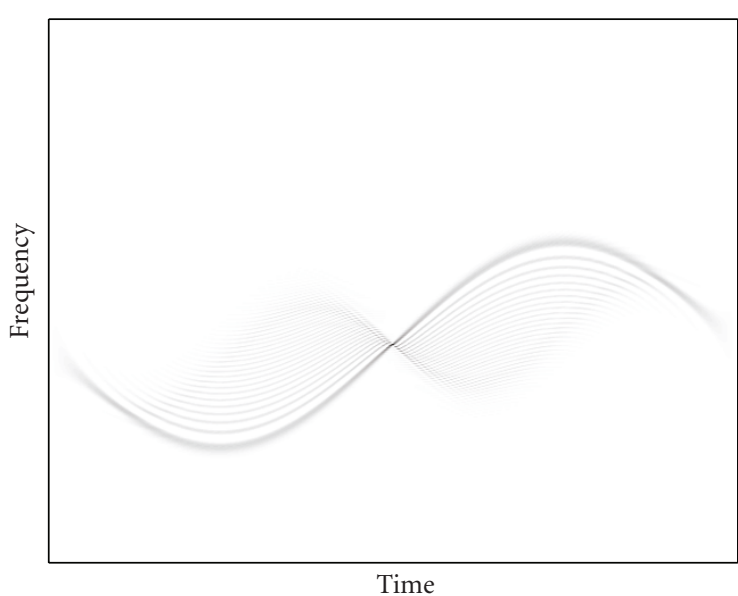

(b)

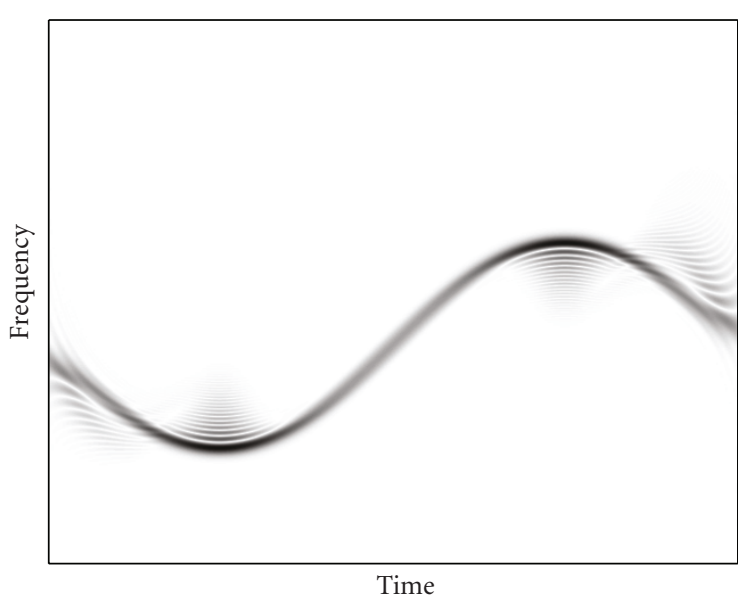

(d)

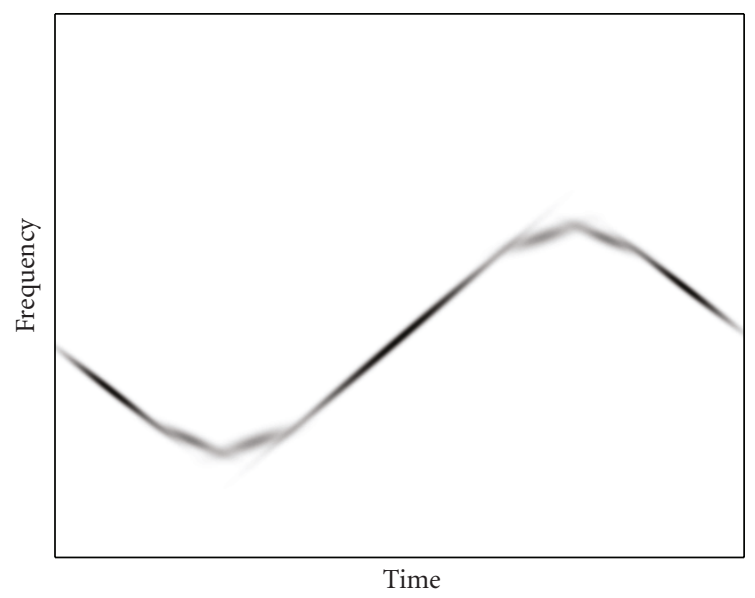

(e)

FIGURE 2: (a) Spectrogram of a frequency-modulated signal. (b) Wigner-Ville representation of the signal (negative energies discarded). (c) Result of proposed method. (d) Choi-Williams representation of the signal. (e) Decomposition of signal in terms of seven chirplets. 


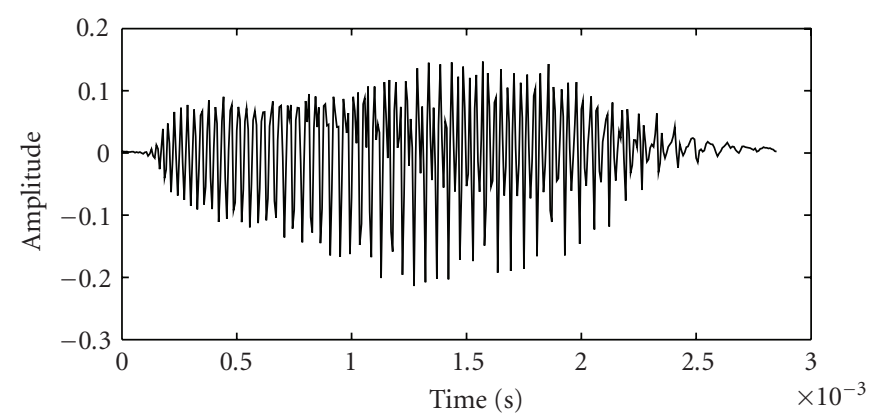

(a)

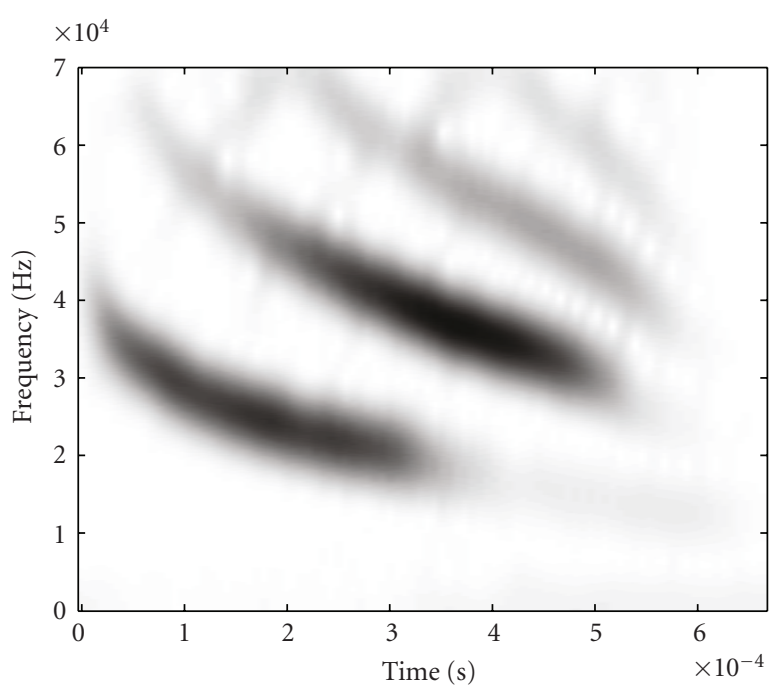

(b)

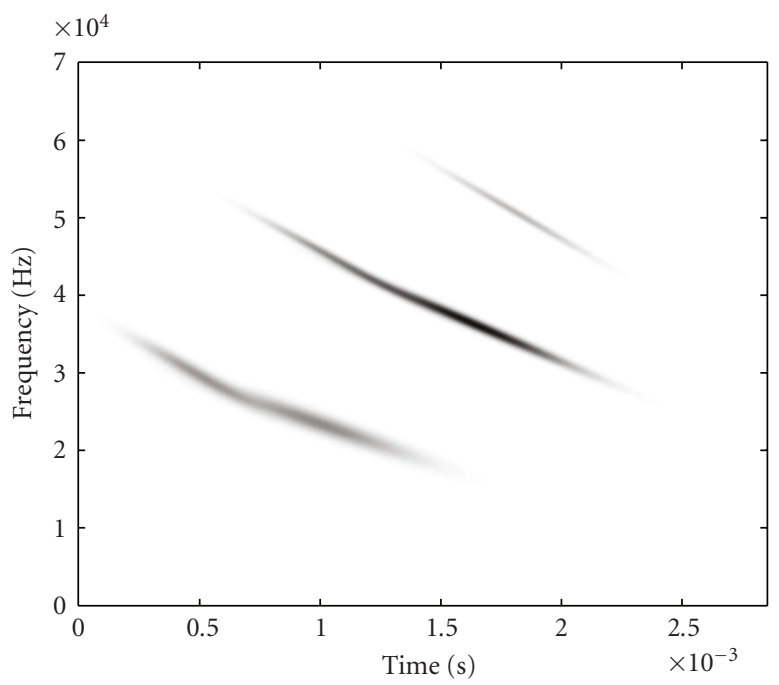

(d)

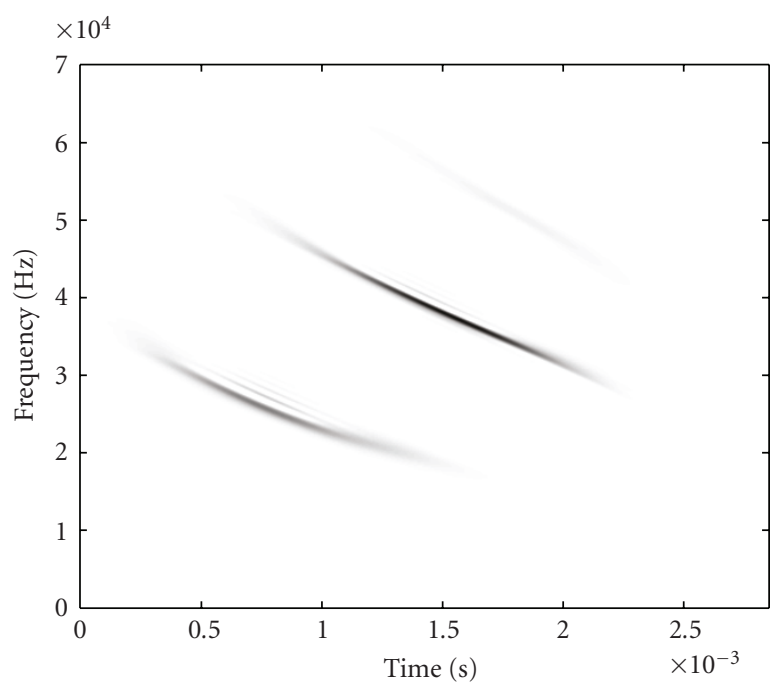

(c)

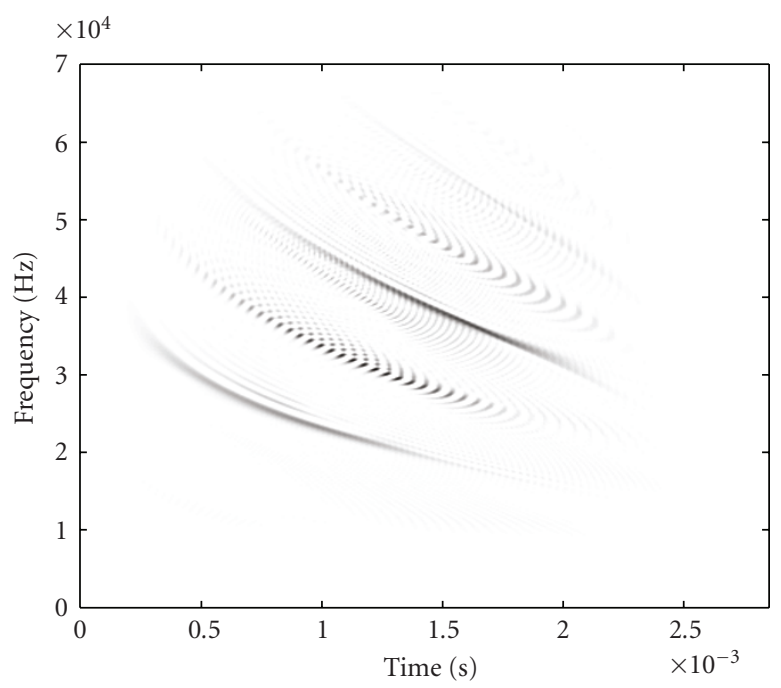

(e)

Figure 3: Chirplet representations of a bioacoustical signal. (a) Time-domain representation of the large brown bat echo-location signal (sampled at $0.14 \mathrm{MHz}$ ). (b) Spectrum of the signal (calculated with a $0.45 \mathrm{~ms}$ Gaussian window). (c) Time-frequency representation of the signal. (d) Chirplet decomposition of the signal (represented by five chirplets). (e) Wigner-Ville distribution of the signal (negative energies discarded). 


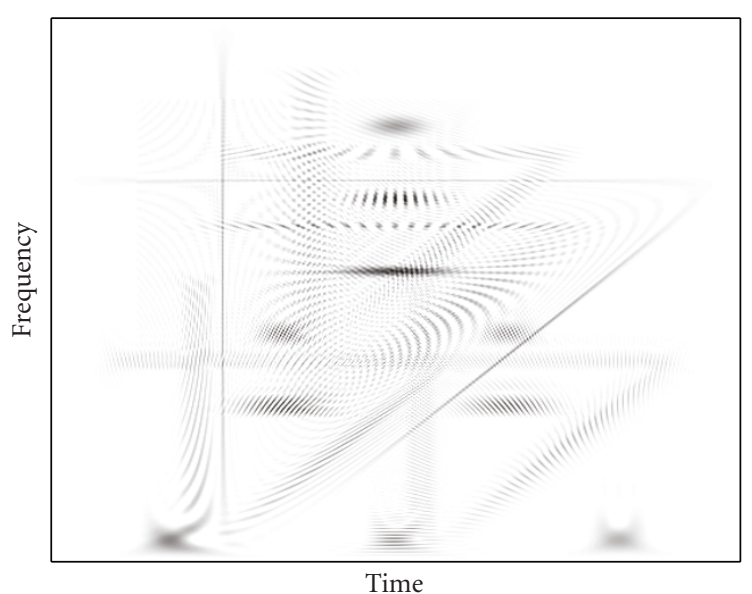

(a)

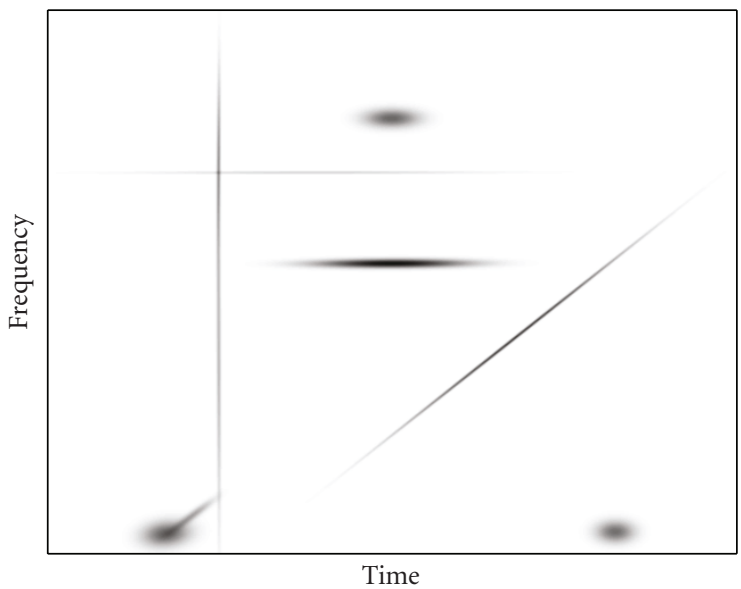

(b)

FIGURE 4: (a) Wigner-Ville distribution of a synthetic signal consisting of a sum of elementary signals [37]. (b) Resulting time-frequency representation.

only in neighbourhood of the origin. This neighbourhood is defined as the circular region around the origin which includes $50 \%$ of the signal energy.

To eliminate artifacts due to sharp cutoffs from kernel filtering (e.g., ringing), the edges of the kernel were smoothed. The smoothing process can be carried out by employing a tapering function like a Hanning or Gaussian function. In Figure 1(d) we show the example of the use of a two-dimensional Gaussian function. A "cleaned" ambiguity representation is then obtained by multiplying the original ambiguity function with the corresponding mask. Finally, the time-frequency representation of the signal is generated by calculating the inverse Fourier transform of the ambiguity function.

If the signal of interest is not a sum of chirplets, the steps outlined above will result in a representation where the signal's energy in the time-frequency plane is approximated by a number of localized straight line segments. It should be noted that for such signals, additional nonchirpletlike interference terms will also appear. These interference terms are often low frequency oscillations that overlap with the signal in ambiguity space. For example, a frequencymodulated signal and its Wigner Ville representation are illustrated in Figure 2. Despite the nature of the signal, the method proposed here can represent the signal in timefrequency space with a high degree of localization. It can be shown that the representation conserves $99 \%$ of the original signal's energy.

The Expectation-Maximization (EM) algorithm was used for finding maximum likelihood estimation of chirplet parameters in the time-frequency plane. All optimization algorithms suffer from difficulties in parameter initialization and in the selection of the number of parameters or components. However, in this case we make use of the parameters estimated from the Radon transformation in ambiguity space. This significiantly reduces the time required for optimization as well as improves the robustness of the estimation. The number of components can also be set equal to the number of peaks found in the Radon transformation, and then adjusting the number of components from there to minimize the total error.

\section{Results and Discussion}

Although biological signals can be found over a wide range of frequencies, they are often narrowband in nature. Chirplets are thus a suitable choice for modelling such signals [37]. The method proposed in this paper uses this property to generate an interference-free time-frequency representation by approximating the underlying time-frequency structures of the signal by a linear approximation. The result provides not only a clearer picture of the salient signal characteristics but also provides a means for mathematically decomposing signals into chirplets. An example of this is shown in Figure 3, where a bat echo-location ultrasound signal is represented as combination of four chirplets. We also show results from synthetically generated signals-see Figure 4 where a signal consisting of a sinusoid, a windowed sinusoid, Gabor logons, sawtooth, an impulse, and a chirplet is analyzed by the same technique. This signal was adapted from [37]. In both cases, the time-frequency visualization is improved significantly and the main time-frequency structures are easily identifiable.

We also provide one example where the time-frequency representation is compared with that which was obtained from chirplet decomposition with matching pursuit. Certain dynamic brain mechanisms can be investigated through neuroelectrical brain responses called event-related potentials (EPRs). The visual evoked potential (VEP) is an evoked brain response generated in the visual cortex in response to the presentation of a visual signal. Such signals are noisy and are often averaged before processing. The VEP signal we have analyzed here is equal to an average of 50 trails from a single subject. Three chirplets are estimated for comparison with the results calculated by Cui and Wong [37] using the matching pursuit algorithm. As can be seen through 


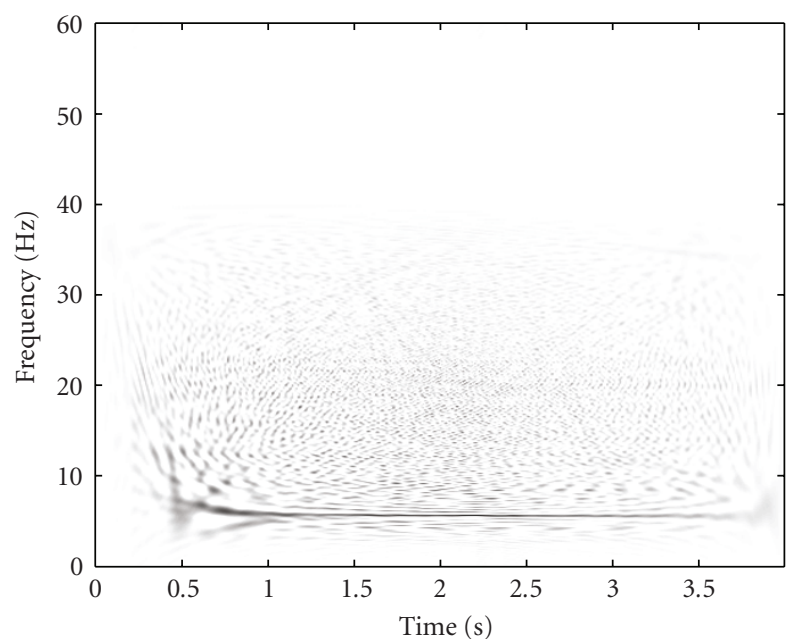

(a)

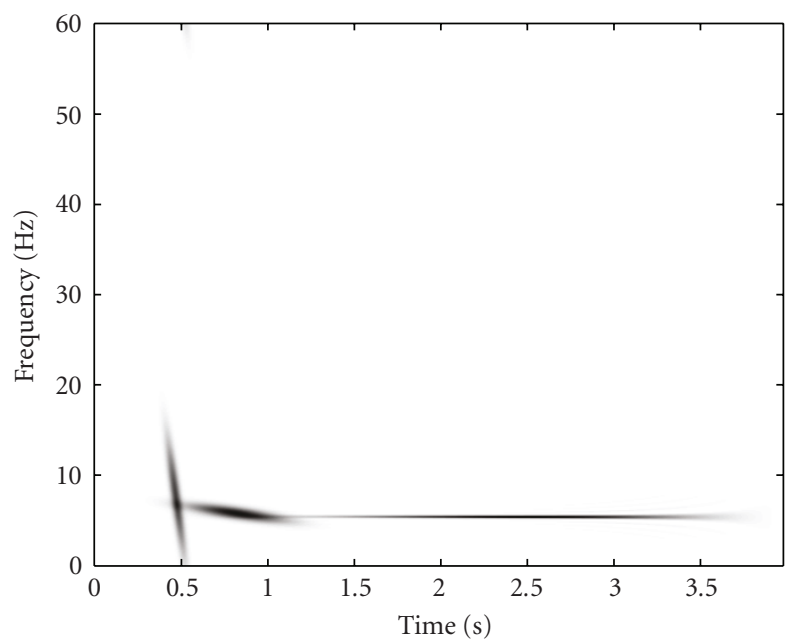

(c)

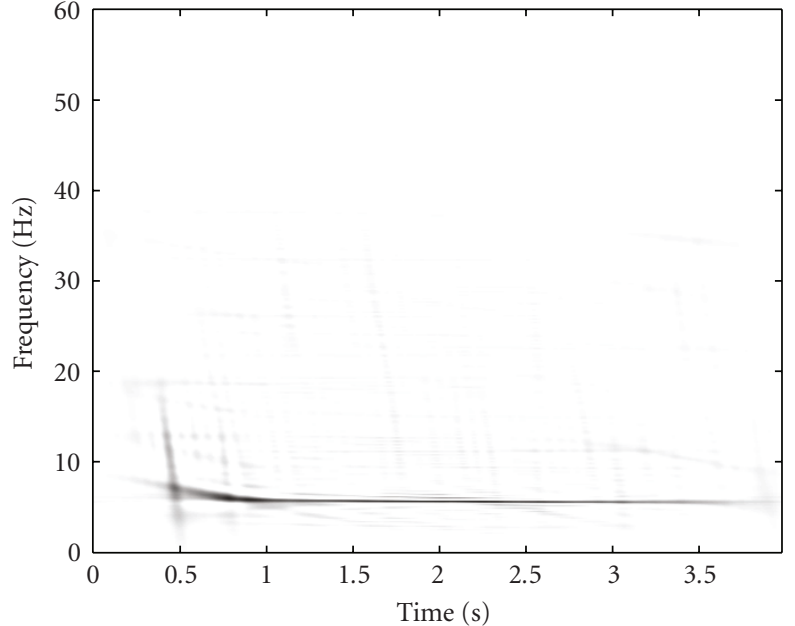

(b)

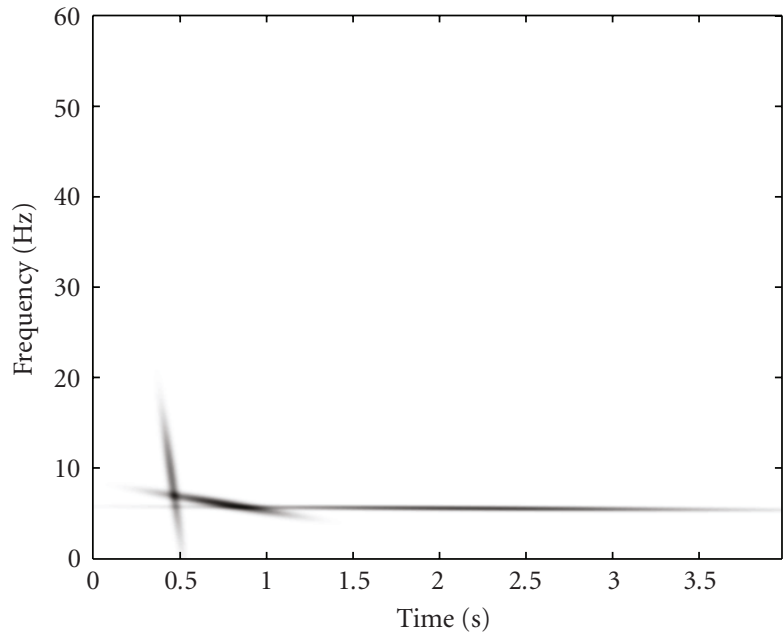

(d)

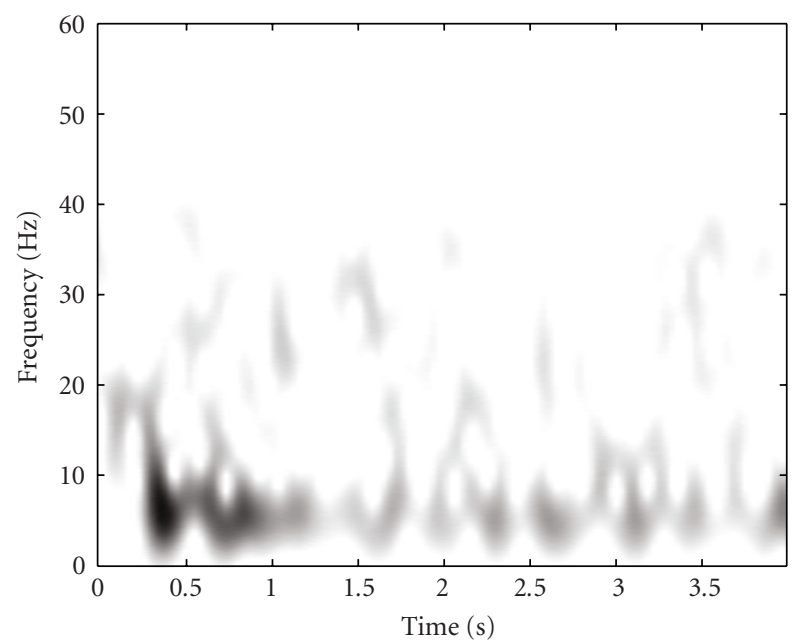

(e)

Figure 5: (a) Wigner-Ville distribution of visual evoked response (negative energies discarded). (b) Resulting signal representation after applying optimal kernel. (c) Result obtained by Cui and Wong through chirplet decomposition via matching pursuit [37]. (d) Three chirplet decomposition by the method proposed here. (e) Spectrogram of corresponding signal. 
comparison of both figures, the results are quite similar. The time-frequency representation was also verified through a spectrogram.

A main challenge for chirplet decomposition is that Gaussian chirplets do not form an orthogonal basis. One solution is to employ suboptimal schemes like matching pursuit. Figure 5 was generated by this particular approach. While the underlying theory of matching pursuit is well established, its numerical implementation in terms of computational speed and accuracy comes at an enormous cost. Matching pursuit requires that a large dictionary of chirplet functions be generated in advance [47]. The signal is decomposed iteratively by finding the best matched dictionary component and then subtracted from the original signal energy. This process continues until the residual energy (error) becomes lower than a specified value. Finding the best projection at each iterative step requires intensive computational processing; maintaining a large dictionary for good resolution and for long-enough signal lengths involves steep storage requirements. In contrast, the method proposed here does not rely on a dictionary and requires far fewer computational steps. Note that chirplet decomposition provides significant data compressibility. The VEP signal shown in Figure 5 consisting of 480 samples can be wellrepresented by as few as 15 parameters in terms of three Gaussian chirplets.

Earlier it was shown that the cross-term interference arising from a pair of monocomponents is located between main components. Moreover, the interferences are oscillatory in nature, and the spatial frequency of these oscillations is a function of the distance between the components in time and frequency. That is, the closer the two components, the lower the oscillation frequency. In ambiguity space, this would mean that the interference lies closer to the origin. The low frequency interference also appears as a result of the signal's instantaneous frequency changing nonlinearly with time. Due to the low frequency nature of these oscillations, the cross-terms may not be completely removed by the kernel due to overlap with signal components in the ambiguity space. Although this interference can be removed at the postprocessing stage by (say) least-squares fitting to a Gaussian density, it is important to remember that this interference contributes to the signal's overall energy distribution.

\section{References}

[1] T. Wang, J. Deng, and B. He, "Classifying EEG-based motor imagery tasks by means of time-frequency synthesized spatial patterns," Clinical Neurophysiology, vol. 115, no. 12, pp. 27442753, 2004.

[2] F. Miwakeichi, E. Martínez-Montes, P. A. Valdés-Sosa, N. Nishiyama, H. Mizuhara, and Y. Yamaguchi, "Decomposing EEG data into space-time-frequency components using Parallel Factor Analysis," NeuroImage, vol. 22, no. 3, pp. 1035-1045, 2004.

[3] B. Aysin, L. F. Chaparro, I. Gravé, and V. Shusterman, "Orthonormal-basis partitioning and time-frequency representation of cardiac rhythm dynamics," IEEE Transactions on Biomedical Engineering, vol. 52, no. 5, pp. 878-889, 2005.
[4] R. Q. Quiroga and H. Garcia, "Single-trial event-related potentials with wavelet denoising," Clinical Neurophysiology, vol. 114, no. 2, pp. 376-390, 2003.

[5] J. Van Zaen, L. Uldry, C. Duchêne et al., "Adaptive tracking of EEG oscillations," Journal of Neuroscience Methods, vol. 186, no. 1, pp. 97-106, 2010.

[6] H.-L. Liu, M.-L. Li, T.-C. Shih et al., "Instantaneous frequency-based ultrasonic temperature estimation during focused ultrasound thermal therapy," Ultrasound in Medicine and Biology, vol. 35, no. 10, pp. 1647-1661, 2009.

[7] E. Merlo, M. Pozzo, G. Antonutto, P. E. Di Prampero, R. Merletti, and D. Farina, "Time-frequency analysis and estimation of muscle fiber conduction velocity from surface EMG signals during explosive dynamic contractions," Journal of Neuroscience Methods, vol. 142, no. 2, pp. 267-274, 2005.

[8] G. Devuyst, J.-M. Vesin, P.-A. Despland, and J. Bogousslavsky, "The matching pursuit: a new method of characterizing microembolic signals?" Ultrasound in Medicine and Biology, vol. 26, no. 6, pp. 1051-1056, 2000.

[9] L. Rankine, N. Stevenson, M. Mesbah, and B. Boashash, "A nonstationary model of newborn EEG," IEEE Transactions on Biomedical Engineering, vol. 54, no. 1, pp. 19-28, 2007.

[10] P. Celka, B. Boashash, and P. Colditz, "Preprocessing and time-frequency analysis of newborn EEG seizures," IEEE Engineering in Medicine and Biology Magazine, vol. 20, no. 5, pp. 30-39, 2001.

[11] A. Monti, C. Médigue, and L. Mangin, "Instantaneous parameter estimation in cardiovascular time series by harmonic and time-frequency analysis," IEEE Transactions on Biomedical Engineering, vol. 49, no. 12 I, pp. 1547-1556, 2002.

[12] A. De Cheveigné, "YIN, a fundamental frequency estimator for speech and music," The Journal of the Acoustical Society of America, vol. 111, no. 4, pp. 1917-1930, 2002.

[13] P. Bonato, S. H. Roy, M. Knaflitz, and C. J. de Luca, "Time frequency parameters of the surface myoelectric signal for assessing muscle fatigue during cyclic dynamic contractions," IEEE Transactions on Biomedical Engineering, vol. 48, no. 7, pp. 745-753, 2001.

[14] I. Shafi, J. Ahmad, S. I. Shah, and F. M. Kashif, "Techniques to obtain good resolution and concentrated time-frequency distributions: a review," EURASIP Journal on Advances in Signal Processing, vol. 2009, 43 pages, 2009.

[15] D. Gabor, "Theory of communication," Journal of the Institute of Electrical Engineers, vol. 93, no. 26, pp. 429-457, 1946.

[16] J. B. Allen and L. R. Rabiner, "A unified approach to short-time fourier analysis and synthesis," Proceedings of the IEEE, vol. 65, no. 11, pp. 1558-1564, 1977.

[17] W. Martin and P. Flandrin, "Wigner-ville spectral analysis of nonstationary processes," IEEE Transactions on Acoustics, Speech, and Signal Processing, vol. 33, no. 6, pp. 1461-1470, 1985.

[18] L. Cohen, "Generalized phase-space distribution functions," Journal of Mathematical Physics, vol. 7, no. 5, pp. 781-786, 1966.

[19] E. Wigner, "On the quantum correction for thermodynamic equilibrium,” Physical Review, vol. 40, no. 5, pp. 749-759, 1932.

[20] H. Margenau and R. N. Hill, "Correlation between measurements in quantum theory," Progress of Theoretical Physics, pp. 772-738, 1961.

[21] T. A. C. M. Claasen and W. F. G. Mecklenbrauker, "Wigner distribution-a tool for time-frequency signal analysis," Philips Journal of Research, vol. 35, no. 4-5, pp. 276-300, 1980. 
[22] P. Flandrin and W. Martin, "A general class of estimators for the wigner-ville spectrum of nonstationary processes," in Systems Analysis and Optimization of Systems, Lecture Notes in Control and Information Sciences, pp. 15-23, Springer, Berlin, Germany, 1984.

[23] R. D. Hippenstiel and P. M. de Oliveira, "Time-varying spectral estimation using the instantaneous power spectrum (IPS)," IEEE Transactions on Acoustics, Speech, and Signal Processing, vol. 38, no. 10, pp. 1752-1759, 1990.

[24] M. Born and P. Jordan, “Zur Quantenmechanik,” Zeitschrift für Physik, vol. 34, no. 1, pp. 858-888, 1925.

[25] H. Choi and W. J. Williams, "Improved time-frequency representation of multicomponent signals using exponential kernels," IEEE Transactions on Acoustics, Speech, and Signal Processing, vol. 37, no. 6, pp. 862-871, 1989.

[26] A. Papandreou and G. F. Boudreaux-Bartels, "Distributions for time-frequency analysis: a generalization of Choi-Williams and the Butterworth distribution," in Proceedings of IEEE International Conference on Acoustics, Speech, and Signal Processing (ICASSP '92), pp. 181-184, San Francisco, Calif, USA, 1992.

[27] J. Jeong and W. J. Williams, "Kernel design for reduced interference distributions," IEEE Transactions on Signal Processing, vol. 40, no. 2, pp. 402-412, 1992.

[28] L. Cohen, "Distributions concentrated along the instantaneous frequency," in Advanced Signal-Processing Algorithms, Architectures, and Implementations, Proceedings of SPIE, pp. 149-157, July 1990.

[29] S. Kadambe, G. F. Boudreaux-Bartels, and P. Duvaut, "Window length selection for smoothing the Wigner distribution by applying an adaptive filter technique," in Proceedings of IEEE International Conference on Acoustics, Speech, and Signal Processing (ICASSP '89), pp. 2226-2229, May 1989.

[30] D. L. Jones and R. G. Baraniuk, "Adaptive optimal-kernel time-frequency representation," IEEE Transactions on Signal Processing, vol. 43, no. 10, pp. 2361-2371, 1995.

[31] J. C. Andrieux, M. R. Feix, G. Mourgues, P. Bertrand, B. Izrar, and V. T. Nguyen, "Optimum smoothing of the wignerville distribution," IEEE Transactions on Acoustics, Speech, and Signal Processing, vol. 35, no. 6, pp. 764-769, 1987.

[32] S. H. Doo, W.-S. Ra, T. S. Yoon, and J. B. Park, "Fast timefrequency domain reflectometry based on the AR coefficient estimation of a chirp signal," in American Control Conference (ACC '09), pp. 3423-3428, June 2009.

[33] M. Jachan, G. Matz, and F. Hlawatsch, "Time-frequency ARMA models and parameter estimators for underspread nonstationary random processes," IEEE Transactions on Signal Processing, vol. 55, no. 9, pp. 4366-4381, 2007.

[34] N. Ma, D. Vray, P. Delachartre, and G. Gimenez, "Timefrequency representation of multicomponent chirp signals," Signal Processing, vol. 56, no. 2, pp. 149-155, 1997.

[35] A. Akan, M. Yalcin, and L. Chaparro, "An iterative method for instantaneous frequency estimation," in Proceedings of the 8th IEEE International Conference on Electronics, Circuits and Systems (ICECS '01), vol. 3, pp. 1335-1338, 2001.

[36] M. Wang, A. K. Chan, and C. K. Chui, "Linear frequencymodulated signal detection using radon-ambiguity transform," IEEE Transactions on Signal Processing, vol. 46, no. 3, pp. 571-586, 1998.

[37] J. Cui and W. Wong, "The adaptive chirplet transform and visual evoked potentials," IEEE Transactions on Biomedical Engineering, vol. 53, no. 7, pp. 1378-1384, 2006.
[38] N. Hess-Nielsen and M. V. Wickerhauser, "Wavelets and timefrequency analysis," Proceedings of the IEEE, vol. 84, no. 4, pp. 523-540, 1996.

[39] S. Mann and S. Haykin, "Chirplet transform: physical considerations," IEEE Transactions on Signal Processing, vol. 43, no. 11, pp. 2745-2761, 1995.

[40] S. Mallat, A Wavelet Tour of Signal Processing, The Sparse Way. Academic Press, London, UK, 3rd edition, 2008.

[41] Y. Lu, R. Demirli, G. Cardoso, and J. Saniie, "A successive parameter estimation algorithm for chirplet signal decomposition," IEEE Transactions on Ultrasonics, Ferroelectrics, and Frequency Control, vol. 53, no. 11, pp. 2121-2131, 2006.

[42] Y. Lu, R. Demirli, G. Cardoso, and J. Saniie, "Chirplet transform for ultrasonic signal analysis and NDE applications," in IEEE Ultrasonics Symposium, pp. 536-539, September 2005.

[43] C. Ioana and A. Quinquis, "On the use of time-frequency warping operators for analysis of marine-mammal signals," in Proceedings of IEEE International Conference on Acoustics, Speech, and Signal Processing (ICASSP '04), pp. 605-608, May 2004.

[44] Y. Tang, X. Luo, and Z. Yang, "Ocean clutter suppression using one-class SVM," in Proceedings of the 14th IEEE Signal Processing Society Workshop on Machine Learning for Signal Processing, pp. 559-568, October 2004.

[45] I. Shafi, J. Ahmad, S. I. Shah, and F. M. Kashif, "Computing de-blurred time frequency distributions using artificial neural networks," Circuits, Systems, and Signal Processing, vol. 27, no. 3, pp. 277-294, 2008.

[46] P. Borgnat and P. Flandrin, "Time-frequency localization from sparsity constraints," in Proceedings of IEEE International Conference on Acoustics, Speech, and Signal Processing (ICASSP '08), pp. 3785-3788, April 2008.

[47] S. G. Mallat and Z. Zhang, "Matching pursuits with timefrequency dictionaries," IEEE Transactions on Signal Processing, vol. 41, no. 12, pp. 3397-3415, 1993. 